\title{
Isozyme analysis of anaerobic rumen fungi and their relationship to aerobic chytrids
}

\author{
Y. W. Ho, ${ }^{1}$ I. Y. S. Khoo, ${ }^{1}$ S. G. Tan, ${ }^{1}$ N. Abdullah, ${ }^{2}$ S. Jalaludin ${ }^{2}$ \\ and $\mathrm{H} . \mathrm{Kudo}^{3}$
}

Author for correspondence: Y. W. Ho. Tel: +603 9486101. Fax: +6039482507.

1,2 Department of Biology ${ }^{1}$
and Department of
Animal Science', Universiti
Pertanian Malaysia,
43400, Serdang, Selangor,
Malaysia
Tropical Agriculture
Research Centre, Tsukuba,
Ibaraki, Japan

\begin{abstract}
Isozymes of $\mathbf{2 3}$ cultures of the anaerobic rumen fungi and seven cultures of aerobic chytridiomycete fungi were analysed by PAGE. A total of 14 isozyme loci were successfully typed by PAGE. They were peptidase A \& C-1, peptidase $A$ \& C-2, peptidase D-1, peptidase D-2, malate dehydrogenase-1, malate dehydrogenase-2, esterase-1, esterase-2, malic enzyme-1, malic enzyme-2, isocitrate dehydrogenase, shikimate dehydrogenase, phosphoglucomutase and 6-phosphogluconate dehydrogenase. Isozyme analysis can be used for studying the genetic relationships among the different anaerobic rumen fungi and the aerobic chytridiomycete fungi and the isozyme characteristics can serve as additional taxonomic criteria in the classification of the anaerobic rumen fungi. A dendrogram based on the isozyme data demonstrated that the anaerobic rumen fungi formed a cluster, indicating a monophyletic group, distinctly separated from the aerobic chytridiomycete fungi. Piromyces communis and $P$. minutus showed a close relationship but $P$. spiralis showed a more distant relationship to both $P$. communis and $P$. minutus. Piromyces as a whole was more related to Caecomyces than to Neocallimastix. Orpinomyces was also found to be more related to Piromyces and Caecomyces than to Neocallimastix. Orpinomyces intercalaris C 70 from cattle showed large genetic variation from 0 . joyonii, indicating that it is a different species.
\end{abstract}

Keywords: Rumen fungi, anaerobic fungi, isozyme analysis, aerobic chytrids, Neocallimastix

\section{INTRODUCTION}

An anaerobic rumen fungus, Neocallimastix frontalis, (Braune) Vavra et Joyon ex Heath, was isolated in 1975 from the rumen contents of sheep (Orpin, 1975). However, its taxonomic status was not established until Heath et al. (1983) studied its ultrastructure, particularly the zoospore ultrastructure, and formally assigned it to the class Chytridiomycetes, order Spizellomycetales and a new family, Neocallimastigaceae [syn. Neocallimasticaceae, $\nmid$ Index of Fungi Supplement (Family Names), 1989] was established to accommodate it. Interest in the anaerobic gut fungi (fungi from the rumen as well as other parts of the gut of herbivores) in recent years has resulted in the discovery and description of many new species (Gold et al., 1988; Barr et al., 1989; Li et al., 1990; Breton et al., 1990, 1991; Ho et al., 1990, 1993a, b, c, 1994).

t The original spelling (Neocallimasticaceae) was corrected to Neocallimastigaceae in the Index of Fungi Supplement (Family Names), 1989.
Many characteristics such as the life cycle, reproductive structures and vegetative thalli of the anaerobic fungi show that they are more closely related to the Chytridiomycetes than to any other group of fungi. Molecular data (18S rRNA sequences) of the fungi also show that they are indeed chytridiomycete fungi (Dore \& Stahl, 1991; Bowman et al., 1992; Li \& Heath, 1992). The classification of the Chytridiomycetes based solely on morphological characteristics has been a matter of contention since many Chytridiomycete species exhibit extensive morphological variation, particularly on different substrates. In view of this, Barr (1980) proposed a new taxonomic system based on thallus development and zoospore ultrastructures which are considered more consistent and reliable. Under this new system, the former order Chytridiales was divided into two orders, namely, the Spizellomycetales Barr and Chytridiales sensu Barr. However, problems still exist for some chytridiomycete fungi which exhibit characteristics of both orders or of different families.

The classification of all the anaerobic gut fungi, studied so 
Table 1. Isozyme allele frequencies in the anaerobic rumen fungi and aerobic chytrids

\begin{tabular}{|c|c|c|c|c|c|c|c|c|c|c|c|c|c|c|c|c|c|c|c|c|}
\hline \multirow[t]{2}{*}{ Locus } & \multirow[t]{2}{*}{ Allele } & \multicolumn{19}{|c|}{ Fungus* } \\
\hline & & $\begin{array}{c}1 \\
(4)\end{array}$ & $\begin{array}{c}2 \\
(5)\end{array}$ & $\begin{array}{c}3 \\
(1)\end{array}$ & $\begin{array}{c}4 \\
\text { (2) }\end{array}$ & $\begin{array}{c}5 \\
(2)\end{array}$ & $\begin{array}{c}6 \\
(2)\end{array}$ & $\begin{array}{c}7 \\
\text { (1) }\end{array}$ & $\begin{array}{c}8 \\
(1)\end{array}$ & $\begin{array}{c}9 \\
(1)\end{array}$ & $\begin{array}{c}10 \\
(1)\end{array}$ & $\begin{array}{c}11 \\
(1)\end{array}$ & $\begin{array}{l}12 \\
\text { (1) }\end{array}$ & $\begin{array}{l}13 \\
(1)\end{array}$ & $\begin{array}{l}14 \\
(1)\end{array}$ & $\begin{array}{l}15 \\
\text { (1) }\end{array}$ & $\begin{array}{l}16 \\
(1)\end{array}$ & $\begin{array}{l}17 \\
(1)\end{array}$ & $\begin{array}{l}18 \\
(1)\end{array}$ & $\begin{array}{l}19 \\
\text { (1) }\end{array}$ \\
\hline \multirow[t]{3}{*}{ PEP A \& C-1 } & 100 & $1 \cdot 0$ & $0 \cdot 80$ & $1 \cdot 0$ & $1 \cdot 0$ & $1 \cdot 0$ & - & - & - & $1 \cdot 0$ & - & $1 \cdot 0$ & $1 \cdot 0$ & $1 \cdot 0$ & - & - & $1 \cdot 0$ & - & - & - \\
\hline & 102 & - & $0 \cdot 20$ & - & - & - & - & - & - & - & - & - & - & - & $1 \cdot 0$ & $1 \cdot 0$ & - & $1 \cdot 0$ & - & - \\
\hline & 0 & -- & - & - & - & - & $1 \cdot 0$ & $1 \cdot 0$ & $1 \cdot 0$ & - & $1 \cdot 0$ & - & - & - & - & - & - & - & $1 \cdot 0$ & $1 \cdot 0$ \\
\hline \multirow[t]{11}{*}{ PEP A \& C-2 } & 100 & - & - & - & - & - & $1 \cdot 0$ & $1 \cdot 0$ & - & $1 \cdot 0$ & - & - & - & - & - & - & - & - & - & - \\
\hline & 102 & - & - & - & 0.5 & $1 \cdot 0$ & - & - & - & - & - & - & - & - & - & - & - & - & - & - \\
\hline & 72 & - & - & - & - & - & - & - & - & - & - & $1 \cdot 0$ & $1 \cdot 0$ & - & - & - & - & - & - & - \\
\hline & 68 & 1.00 & 0.80 & $1 \cdot 0$ & - & - & - & - & - & - & - & - & - & - & - & - & - & - & - & - \\
\hline & 64 & - & 0.20 & - & - & - & - & - & - & - & - & - & - & - & - & - & - & - & - & - \\
\hline & 60 & - & - & - & - & - & - & - & - & - & - & - & - & - & - & - & - & - & - & $1 \cdot 0$ \\
\hline & 56 & - & - & - & - & - & - & - & - & - & - & - & - & - & - & - & - & - & $1 \cdot 0$ & - \\
\hline & 52 & - & - & - & - & - & - & - & - & - & - & - & - & - & $1 \cdot 0$ & - & $1 \cdot 0$ & - & - & - \\
\hline & 50 & - & - & - & - & - & - & - & - & - & - & - & - & - & - & - & - & 1.0 & - & - \\
\hline & 46 & - & - & - & - & - & - & - & - & - & - & - & - & $1 \cdot 0$ & - & 1.0 & - & - & - & - \\
\hline & 0 & - & - & - & 0.50 & - & - & - & 1.0 & - & $1 \cdot 0$ & - & - & - & - & - & - & - & - & - \\
\hline \multirow[t]{5}{*}{ PEP D-1 } & 100 & - & - & - & - & - & - & - & - & $1 \cdot 0$ & - & - & - & - & - & - & - & - & - & - \\
\hline & 106 & $0 \cdot 25$ & - & - & - & - & - & - & - & - & - & - & - & - & - & - & - & - & - & - \\
\hline & 104 & - & - & - & 0.5 & - & - & - & - & - & - & - & - & - & $1 \cdot 0$ & $1 \cdot 0$ & - & - & - & - \\
\hline & 102 & 0.75 & $0 \cdot 80$ & $1 \cdot 0$ & - & - & - & - & - & - & - & - & - & $1 \cdot 0$ & - & - & $1 \cdot 0$ & - & $1 \cdot 0$ & $1 \cdot 0$ \\
\hline & 0 & - & 0.20 & - & 0.5 & $1 \cdot 0$ & $1 \cdot 0$ & $1 \cdot 0$ & $1 \cdot 0$ & - & $1 \cdot 0$ & $1 \cdot 0$ & $1 \cdot 0$ & - & - & - & - & $1 \cdot 0$ & - & - \\
\hline \multirow[t]{2}{*}{ PEP D-2 } & 100 & - & - & - & $0 \cdot 5$ & - & - & - & - & $1 \cdot 0$ & $1 \cdot 0$ & - & - & - & - & - & - & $1 \cdot 0$ & $1 \cdot 0$ & $1 \cdot 0$ \\
\hline & 0 & $1 \cdot 0$ & $1 \cdot 0$ & $1 \cdot 0$ & $0 \cdot 5$ & $1 \cdot 0$ & $1 \cdot 0$ & $1 \cdot 0$ & $1 \cdot 0$ & - & - & $1 \cdot 0$ & $1 \cdot 0$ & $1 \cdot 0$ & $1 \cdot 0$ & $1 \cdot 0$ & $1 \cdot 0$ & - & - & - \\
\hline \multirow[t]{5}{*}{ MDH-1 } & 100 & 0.5 & $1 \cdot 0$ & $1 \cdot 0$ & $1 \cdot 0$ & $1 \cdot 0$ & $1 \cdot 0$ & $1 \cdot 0$ & $1 \cdot 0$ & $1 \cdot 0$ & $1 \cdot 0$ & $1 \cdot 0$ & $1 \cdot 0$ & $1 \cdot 0$ & - & - & - & - & - & - \\
\hline & 106 & - & - & - & - & - & - & - & - & - & - & - & - & - & - & $1 \cdot 0$ & - & - & - & - \\
\hline & 98 & 0.5 & - & - & - & - & - & - & - & - & - & - & - & - & - & - & - & - & - & - \\
\hline & 96 & - & - & - & - & - & - & - & - & - & - & - & - & - & - & $1 \cdot 0$ & - & $1 \cdot 0$ & $1 \cdot 0$ & $1 \cdot 0$ \\
\hline & 0 & - & - & - & - & - & - & - & - & - & - & - & - & - & $1 \cdot 0$ & - & - & - & - & - \\
\hline \multirow[t]{11}{*}{ MDH-2 } & 100 & - & - & - & - & - & - & - & - & $1 \cdot 0$ & - & - & - & - & - & - & - & - & - & - \\
\hline & 102 & - & - & - & $0 \cdot 5$ & - & - & - & - & - & $1 \cdot 0$ & - & - & - & - & - & - & - & - & - \\
\hline & 96 & - & - & - & - & - & - & - & - & - & - & $1 \cdot 0$ & - & - & - & - & - & - & - & - \\
\hline & 94 & - & - & - & - & - & - & - & - & - & - & - & $1 \cdot 0$ & - & - & - & - & $1 \cdot 0$ & - & - \\
\hline & 92 & - & 0.2 & - & - & - & - & - & - & - & - & - & - & - & - & - & - & - & - & - \\
\hline & 90 & $1 \cdot 0$ & 0.8 & $1 \cdot 0$ & - & - & - & - & - & - & - & - & - & - & - & - & - & - & - & - \\
\hline & 88 & - & - & - & 0.5 & 1.0 & - & - & - & - & - & - & - & - & - & - & - & - & - & - \\
\hline & 86 & - & - & - & - & - & $1 \cdot 0$ & $1 \cdot 0$ & $1 \cdot 0$ & - & - & - & - & - & - & - & - & - & - & - \\
\hline & 82 & - & - & - & - & - & - & - & - & - & - & - & - & - & - & 1.0 & - & - & - & - \\
\hline & 76 & - & - & - & - & - & - & - & - & - & - & - & - & $1 \cdot 0$ & - & - & - & - & - & - \\
\hline & 0 & - & - & - & - & - & - & - & - & - & - & - & - & - & $1 \cdot 0$ & - & $1 \cdot 0$ & - & $1 \cdot 0$ & $1 \cdot 0$ \\
\hline \multirow[t]{4}{*}{ ES-1 } & 100 & $1 \cdot 0$ & $1 \cdot 0$ & $1 \cdot 0$ & - & - & - & - & - & - & - & - & - & - & - & - & - & - & - & - \\
\hline & 88 & - & - & - & - & - & - & - & - & - & - & $1 \cdot 0$ & - & - & - & - & - & - & - & - \\
\hline & 60 & - & - & - & - & - & - & - & - & - & - & - & $1 \cdot 0$ & - & - & - & - & - & - & - \\
\hline & 0 & - & - & - & $1 \cdot 0$ & $1 \cdot 0$ & $1 \cdot 0$ & $1 \cdot 0$ & $1 \cdot 0$ & $1 \cdot 0$ & $1 \cdot 0$ & - & - & $1 \cdot 0$ & $1 \cdot 0$ & $1 \cdot 0$ & $1 \cdot 0$ & $1 \cdot 0$ & $1 \cdot 0$ & $1 \cdot 0$ \\
\hline \multirow[t]{2}{*}{ ES-2 } & 100 & $1 \cdot 0$ & $1 \cdot 0$ & $1 \cdot 0$ & - & - & - & - & - & - & - & - & - & - & - & - & - & - & - & - \\
\hline & 0 & - & - & - & $1 \cdot 0$ & $1 \cdot 0$ & $1 \cdot 0$ & $1 \cdot 0$ & $1 \cdot 0$ & $1 \cdot 0$ & $1 \cdot 0$ & $1 \cdot 0$ & $1 \cdot 0$ & $1 \cdot 0$ & 1.0 & $1 \cdot 0$ & $1 \cdot 0$ & $1 \cdot 0$ & $1 \cdot 0$ & $1 \cdot 0$ \\
\hline \multirow[t]{9}{*}{ ME-1 } & 100 & - & $0 \cdot 2$ & $1 \cdot 0$ & 0.5 & - & - & - & - & $1 \cdot 0$ & $1 \cdot 0$ & 1.0 & $1 \cdot 0$ & - & - & - & - & - & - & - \\
\hline & 96 & $1 \cdot 0$ & $0 \cdot 6$ & - & - & - & - & - & - & - & - & - & - & $1 \cdot 0$ & - & - & - & - & - & - \\
\hline & 92 & - & $1 \cdot 0$ & - & - & - & - & - & - & - & - & - & - & - & - & - & - & - & - & - \\
\hline & 88 & - & - & - & 0.5 & $1 \cdot 0$ & $1 \cdot 0$ & 1.0 & 1.0 & - & - & - & - & - & - & - & - & - & - & - \\
\hline & 82 & - & - & - & - & - & - & - & - & - & - & - & - & $1 \cdot 0$ & - & - & - & - & - & - \\
\hline & 74 & - & - & - & - & - & - & -- & - & - & - & - & - & - & - & - & - & - & - & $1 \cdot 0$ \\
\hline & 68 & - & - & - & - & - & - & -- & - & - & - & - & - & - & - & - & $1 \cdot 0$ & - & - & - \\
\hline & 50 & - & - & - & - & - & - & ..- & - & - & - & - & - & - & - & $1 \cdot 0$ & - & $1 \cdot 0$ & - & - \\
\hline & 0 & - & - & - & - & - & - & .-- & - & - & - & - & - & - & - & - & - & - & $1 \cdot 0$ & - \\
\hline
\end{tabular}


Table 1 (cont.)

\begin{tabular}{|c|c|c|c|c|c|c|c|c|c|c|c|c|c|c|c|c|c|c|c|c|}
\hline \multirow[t]{2}{*}{ Locus } & \multirow[t]{2}{*}{ Allele } & \multicolumn{19}{|c|}{ Fungus* } \\
\hline & & $\begin{array}{c}1 \\
(4)\end{array}$ & $\begin{array}{c}2 \\
(5)\end{array}$ & $\begin{array}{c}3 \\
(1)\end{array}$ & $\begin{array}{c}4 \\
(2)\end{array}$ & $\begin{array}{c}5 \\
(2)\end{array}$ & $\begin{array}{c}6 \\
(2)\end{array}$ & $\begin{array}{c}7 \\
(1)\end{array}$ & $\begin{array}{c}8 \\
(1)\end{array}$ & $\begin{array}{c}9 \\
(1)\end{array}$ & $\begin{array}{l}10 \\
(1)\end{array}$ & $\begin{array}{l}11 \\
(1)\end{array}$ & $\begin{array}{l}12 \\
(1)\end{array}$ & $\begin{array}{l}13 \\
\text { (1) }\end{array}$ & $\begin{array}{l}14 \\
(1)\end{array}$ & $\begin{array}{l}15 \\
(1)\end{array}$ & $\begin{array}{l}16 \\
(1)\end{array}$ & $\begin{array}{c}17 \\
\text { (1) }\end{array}$ & $\begin{array}{l}18 \\
\text { (1) }\end{array}$ & $\begin{array}{l}19 \\
\text { (1) }\end{array}$ \\
\hline \multirow[t]{12}{*}{$\mathrm{ME}-2$} & 100 & 0.4 & $1 \cdot 0$ & - & - & - & - & - & - & $1 \cdot 0$ & - & - & $1 \cdot 0$ & - & - & - & - & $1 \cdot 0$ & - & - \\
\hline & 116 & - & - & - & - & - & - & - & - & - & - & - & - & - & - & - & $1 \cdot 0$ & - & - & - \\
\hline & 114 & - & $0 \cdot 2$ & - & 0.5 & - & - & - & - & - & - & - & - & - & - & - & - & - & - & - \\
\hline & 106 & - & - & - & - & - & - & - & - & - & - & 1.0 & - & - & - & - & - & - & - & - \\
\hline & 92 & $1 \cdot 0$ & 0.4 & - & - & - & - & - & - & - & $1 \cdot 0$ & - & - & - & - & - & - & - & - & - \\
\hline & 90 & - & - & - & $0 \cdot 5$ & $1 \cdot 0$ & - & - & - & - & - & - & - & - & - & - & - & - & - & - \\
\hline & 88 & - & - & - & - & - & $0 \cdot 15$ & - & $1 \cdot 0$ & - & - & - & - & - & - & - & - & - & - & - \\
\hline & 86 & - & - & - & - & - & 0.5 & $1 \cdot 0$ & - & - & - & - & - & - & - & - & - & - & - & - \\
\hline & 82 & - & - & - & - & - & - & - & - & - & - & - & - & - & $1 \cdot 0$ & - & - & - & - & - \\
\hline & 66 & - & - & - & - & - & - & - & - & - & - & - & - & - & - & $1 \cdot 0$ & - & - & - & - \\
\hline & 58 & - & - & - & - & - & - & - & - & - & - & - & - & $1 \cdot 0$ & - & - & - & - & - & - \\
\hline & 0 & - & - & - & - & - & - & - & - & - & - & - & - & - & - & - & - & - & $1 \cdot 0$ & $1 \cdot 0$ \\
\hline \multirow[t]{8}{*}{ IDH } & 100 & - & - & - & - & - & - & - & - & $1 \cdot 0$ & - & - & - & - & - & - & - & - & - & - \\
\hline & 108 & - & $0 \cdot 2$ & - & - & - & - & - & - & - & $1 \cdot 0$ & - & - & - & - & - & - & - & - & - \\
\hline & 102 & $1 \cdot 0$ & 0.4 & - & - & - & - & - & - & - & - & - & - & - & - & - & - & - & - & - \\
\hline & 98 & - & 0.2 & $1 \cdot 0$ & - & - & - & - & - & - & - & - & - & - & - & - & -- & - & - & - \\
\hline & 96 & - & - & - & - & - & 0.5 & - & $1 \cdot 0$ & - & - & - & - & - & - & - & - & - & - & - \\
\hline & 92 & - & $0 \cdot 2$ & - & 0.5 & 0.5 & - & $1 \cdot 0$ & - & - & - & $1 \cdot 0$ & - & - & - & - & - & - & - & - \\
\hline & 90 & - & - & - & - & 0.5 & - & - & - & - & - & - & - & - & - & - & - & - & - & - \\
\hline & 0 & - & - & - & 0.5 & - & 0.5 & - & - & - & - & - & $1 \cdot 0$ & $1 \cdot 0$ & 1.0 & $1 \cdot 0$ & $1 \cdot 0$ & $1 \cdot 0$ & $1 \cdot 0$ & 1.0 \\
\hline \multirow[t]{3}{*}{ SkDH } & 100 & $1 \cdot 0$ & $0 \cdot 8$ & $1 \cdot 0$ & $1 \cdot 0$ & $1 \cdot 0$ & $1 \cdot 0$ & $1 \cdot 0$ & $1 \cdot 0$ & $1 \cdot 0$ & $1 \cdot 0$ & - & - & - & $1 \cdot 0$ & $1 \cdot 0$ & $1 \cdot 0$ & $1 \cdot 0$ & $1 \cdot 0$ & - \\
\hline & 112 & - & - & - & - & - & - & - & - & - & - & - & - & $1 \cdot 0$ & - & - & - & - & - & - \\
\hline & 98 & - & 0.2 & - & - & - & - & - & - & - & - & $1 \cdot 0$ & $1 \cdot 0$ & - & - & - & - & - & - & - \\
\hline \multirow[t]{7}{*}{ PGM } & 100 & - & - & - & - & - & - & - & - & $1 \cdot 0$ & - & - & - & - & - & - & - & - & - & - \\
\hline & 96 & - & - & - & - & - & - & - & - & - & - & $1 \cdot 0$ & - & - & - & - & - & - & - & - \\
\hline & 94 & - & - & - & - & - & 0.5 & - & - & - & - & - & - & - & - & - & - & - & - & - \\
\hline & 92 & $0 \cdot 75$ & 0.8 & $1 \cdot 0$ & - & - & - & - & - & - & - & - & - & - & - & -- & - & - & - & - \\
\hline & 74 & - & - & - & - & - & - & - & - & - & - & - & - & - & - & - & $1 \cdot 0$ & - & - & - \\
\hline & 70 & - & - & - & - & - & - & - & - & - & - & - & - & - & - & - & - & - & - & 1.0 \\
\hline & 0 & $0 \cdot 25$ & 0.2 & - & $1 \cdot 0$ & $1 \cdot 0$ & 0.5 & 1.0 & $1 \cdot 0$ & - & 1.0 & - & $1 \cdot 0$ & $1 \cdot 0$ & $1 \cdot 0$ & 1.0 & - & $1 \cdot 0$ & $1 \cdot 0$ & - \\
\hline \multirow[t]{4}{*}{ 6-PGDH } & 100 & - & - & - & - & - & - & - & - & - & - & - & - & $1 \cdot 0$ & $1 \cdot 0$ & - & $1 \cdot 0$ & $1 \cdot 0$ & - & - \\
\hline & 110 & - & - & - & - & - & - & - & - & - & - & - & - & - & - & - & - & - & $1 \cdot 0$ & - \\
\hline & 102 & - & - & - & - & - & - & - & - & - & - & - & - & - & - & $1 \cdot 0$ & - & - & - & - \\
\hline & 0 & $1 \cdot 0$ & $1 \cdot 0$ & $1 \cdot 0$ & $1 \cdot 0$ & $1 \cdot 0$ & $1 \cdot 0$ & $1 \cdot 0$ & $1 \cdot 0$ & $1 \cdot 0$ & $1 \cdot 0$ & $1 \cdot 0$ & $1 \cdot 0$ & - & - & - & - & - & - & $1 \cdot 0$ \\
\hline
\end{tabular}

* 1 , N. variabilis (buffalo); 2, N. variabilis (cattle); 3, Neocallimastix sp. C148; 4, P. communis (goat); $5, P$. communis (sheep); 6, P. minutus (goat); 7, P. minutus (sheep); 8, P. minutus (deer); 9, P. spiralis (goat); 10, Caecomyces sp. (buffalo); 11, Orpinomyces joyonii (buffalo); 12, Orpinomyces intercalaris $\mathrm{C} 70$ (cattle); 13, S. plurigibbosus BR 33;14, Chytridium confervae BR 97; 15, S. punctatus BR 117; 16, R. rosea BR 60;17, S. acuminatus BR $62 ; 18$, R. rosea BR 186; 19, Catenaria anguillulae BR 105. Numbers in parentheses indicate the number of isolates used.

far, is based mainly on the morphological and ultrastructural characteristics. As morphological characteristics may vary under different cultural conditions, other characteristics such as biochemical and genetic properties could be used as additional taxonomic criteria. Dore \& Stahl (1991), Li \& Heath (1992) and Li et al. (1993) assessed the phylogenetic relationships among the anaerobic gut fungi and their relationships with other eukaryotes using $18 \mathrm{~S}$ rRNA sequences and cladistic analysis of structural data and showed that the anaerobic gut fungi are monophyletic and form a closely related group in the Chytridiomycetes. However, the gut fungi were found to be distinct from both the Spizellomycetales and the Chytridiales and a new order, the Neocallimastigales [syn. Neocallimasticales, Index of Fungi Supplement (Family Names), 1989] was established to accommodate them (Li et al., 1993). At the generic level, the phylogenetic relationships among Neocallimastix, Piromyces and Orpinomyces are still controversial (Li \& Heath, 1992; Li et al., 1993) and need further analyses. 
The genetic relationships of different taxa can also be assessed by comparing their isozymes. The objective of the present investigation was to determine the relationships among the anaerobic rumen fungi and their relationships to the aerobic chytridiomycete fungi based on their isozyme characteristics.

\section{METHODS}

Fungal isolates used. Twenty-three isolates of anaerobic rumen fungi and seven isolates of aerobic chytridiomycete fungi were studied. The anaerobic rumen fungal isolates, obtained from the rumen of fistulated animals, were Neocallimastix variabilis Ho [four isolates from buffalo (Bubalus bubalis), five isolates from cattle (Bos indicus), including isolate C 15 which is the type culture for N. variabilis], one isolate, C 148 of Neocallimastix sp. from cattle, five isolates of Piromyces minutus Ho [two isolates from goat (Capra bircus), two from sheep (Ovis aries) and the type culture, D 2, from deer (Cervus nippon)], four isolates of $P$. communis Gold (two cultures from sheep and two from goat), one isolate of $P$. spiralis $\mathrm{Ho}$ (the type culture, G 34, from goat), one isolate of Caecomyces sp. (from buffalo), one isolate of Orpinomyces joyonii (Breton et al.) Li et al. (B 48 from buffalo) and one isolate of $O$. intercalaris $\mathrm{Ho} \mathrm{sp}$. (C 70, from cattle). When the fungal species are represented by more than one isolate from a particular host, the isolates were obtained from separate animals on different occasions.

The seven aerobic chytridiomycete fungal isolates were obtained from Dr D. J. S. Barr, Centre for Land and Biological Resources Research, Central Experimental Farm, Research Branch, Agriculture Canada, Ottawa, Ontario, Canada. The isolates (one each) were Spizellomyces plurigibbosus Barr (BR 33), S. acuminatus Barr (BR 62), S. punctatus (Koch) Barr (BR 117), Rbizophlyctis rosea (de Bary \& Woronin) Fischer (BR 60), R. rosea (BR 186), Chytridium confervae (Wille) Minden (BR 97) and Catenaria anguillulae Sorokin (BR 105). Spizellomyces and Rbizophlyctis belong to the order Spizellomycetales, Chytridium confervae to the order Chytridiales and Catenaria anguillulae to the order Blastocladiales.

Culturing fungal isolates for routine maintenance and for morphological studies. The media for culturing the anaerobic rumen fungal isolates for morphological studies were glucose sloppy medium, cellulose sloppy medium (Ho \& Bauchop, 1991) and liquid medium (Barr et al., 1989) with straw as the carbon source. The fungal isolates were grown anaerobically (Hungate, 1969) at $39^{\circ} \mathrm{C}$ in roll tubes containing 7.0 ml medium.

The aerobic chytridiomycete fungi were cultured in petri dishes ( $9 \mathrm{~cm}$ diameter) containing $15 \mathrm{ml}$ yeast-peptone-glucose agar. The medium was similar to that described by Barr (1984). The cultures were incubated at $28 \pm 1^{\circ} \mathrm{C}$.

Morphological characteristics of both the anaerobic rumen fungi and the aerobic chytridiomycete fungi were observed daily for 3-4 d using bright-field and phase-contrast microscopy.

Culturing fungal isolates for electrophoresis. The medium used for culturing the anaerobic rumen fungi for electrophoresis was glucose broth medium which was glucose sloppy medium without agar. The medium was prepared under anaerobic conditions using techniques described by Hungate (1969), and $500 \mathrm{ml}$ and $1 \mathrm{l}$ of the medium were dispensed into $500 \mathrm{ml}$ and 11 Duran bottles (Schott), respectively. The bottles were closed tightly with screw caps. Ten replicates were made for each medium volume and the bottles were autoclaved for $30 \mathrm{~min}$ at $121^{\circ} \mathrm{C}$ and $1.4 \mathrm{~kg} \mathrm{~cm}^{-1}$. When cooled, the bottles were inocu- lated with $1 \%(\mathrm{v} / \mathrm{v})$ fungal inoculum and incubated at $39^{\circ} \mathrm{C}$ for $3 \mathrm{~d}$ after which the fungal thalli were harvested.

The aerobic chytridiomycete fungi were cultured in $250 \mathrm{ml}$ Erlenmeyer flasks containing $100 \mathrm{ml}$ yeast-peptone-glucose liquid medium. The medium was similar to yeast-peptoneglucose agar except that agar was omitted. The medium was autoclaved and inoculated in the same manner as described above. The culture was incubated at $28 \pm 1{ }^{\circ} \mathrm{C}$ for $3 \mathrm{~d}$ after which the fungal thalli were harvested for electrophoresis.

Harvesting of fungal thalli for electrophoresis. Neocallimastix variabilis, $O$. joyonii and $O$. intercalaris $\mathrm{sp}$. which produced thalli that were quite large were harvested by filtering through a Buchner funnel lined with a nylon cloth (mesh size $20 \mu \mathrm{m}$ ). Spizellomyces acuminatus (BR 62) which produced small thalli of low density was harvested by microfiltration through a layer of cellulose nitrate filter paper with a pore size of $0.45 \mu \mathrm{m}$ diameter. All the other fungal isolates were harvested by centrifuging twice at $30000 \mathrm{~g}$ for $40 \mathrm{~min}$ at $4{ }^{\circ} \mathrm{C}$. All the harvested fungal thalli were washed three times with distilled water and stored immediately in liquid nitrogen at $-196^{\circ} \mathrm{C}$. The stored fungal thalli were used for enzyme extraction within a week.

Extraction of enzyme. About $0.2 \mathrm{~g}$ of frozen fungal thalli taken from liquid nitrogen storage was ground into a fine powder using an ice-cold mortar and pestle and $0.3 \mathrm{ml}$ extraction buffer, $0 \cdot 1 \mathrm{M}$ Tris/ $\mathrm{HCl}, \mathrm{pH} 7 \cdot 1$, was added. The extract was then transferred into ampoules and centrifuged at $20000 \mathrm{~g}$ for $40 \mathrm{~min}$ at $4^{\circ} \mathrm{C}$. The supernatant was used for electrophoresis.

PAGE. PAGE was carried out using the $7 \%$ horizontal gel procedure described by Tan \& Teng (1979). Twenty-four enzyme systems were studied using CA-7 buffer (Steiner \& Joslyn, 1979). They were alcohol dehydrogenase (ADH) EC 1.1.1.1, peptidase A \& $C$ (Leu-Ala) (PEP A \& C) EC 3.4.11, peptidase D (Phe-Pro) (PEP D) EC 3.4.13.9, succinate dehydrogenase $(\mathrm{SuDH})$ EC 1.3.99.1, lactate dehydrogenase (LDH) EC 1.1.1.27, aconitase (ACO) EC 4.2.1.3, $\beta$-hydroxybutyrate dehydrogenase $(\mathrm{HBDH})$ EC 1.1.1.30, xanthine dehydrogenase (XDH) EC 1.1.1.204, sorbitol dehydrogenase (SORDH) EC 1.1.1.14, glutamate dehydrogenase (GLD) EC 1.4.1.2, glutamate oxaloacetate transaminase (GOT) EC 2.6.1.1, hexokinase (HK) EC 2.7.1.1, acid phosphatase (ACP) EC 3.1.3.2, glucose dehydrogenase (GDH) EC 1.1.1.47, glucose-6-phosphate dehydrogenase (G6PD) EC 1.1.1.49, shikimate dehydrogenase (SkDH) EC 1.1.1.25, isocitrate dehydrogenase (IDH) EC 1.1.1.42, phosphoglucoisomerase (PGI) EC 5.3.1.9, malic enzyme (ME) EC 1.1.1.40, mannose-6-phosphate isomerase (MPI) EC 5.3.1.8, malate dehydrogenase (MDH) EC 1.1.1.37, phosphoglucomutase (PGM) EC 5.4.2.2, 6phosphogluconate dehydrogenase (6-PGDH) EC 1.1.1.44 and carboxylesterase (ES) EC 3.1.1.1.

The staining procedures for the various enzymes were adapted from Harris \& Hopkinson (1976) and Vallejos (1983). The stained gels were rinsed with a wash solution containing $250 \mathrm{ml}$ methanol, $100 \mathrm{ml}$ glacial acetic acid and $650 \mathrm{ml}$ distilled water. The isozyme banding patterns were observed directly from the gel which was placed on a light box and scored. The BIOsys-1 computer package of Swofford \& Selander (1989) was used to analyse the isozyme data. The experiments were done twice. All the chemicals used for enzyme extraction and PAGE were from Sigma.

Nei's unbiased genetic identity $(I)$ (Nei, 1978) was used to estimate the proportion of genes that are identical in structure in any two populations of fungi. For fungal species with more than one isolate from an animal species, the isolates were analysed 


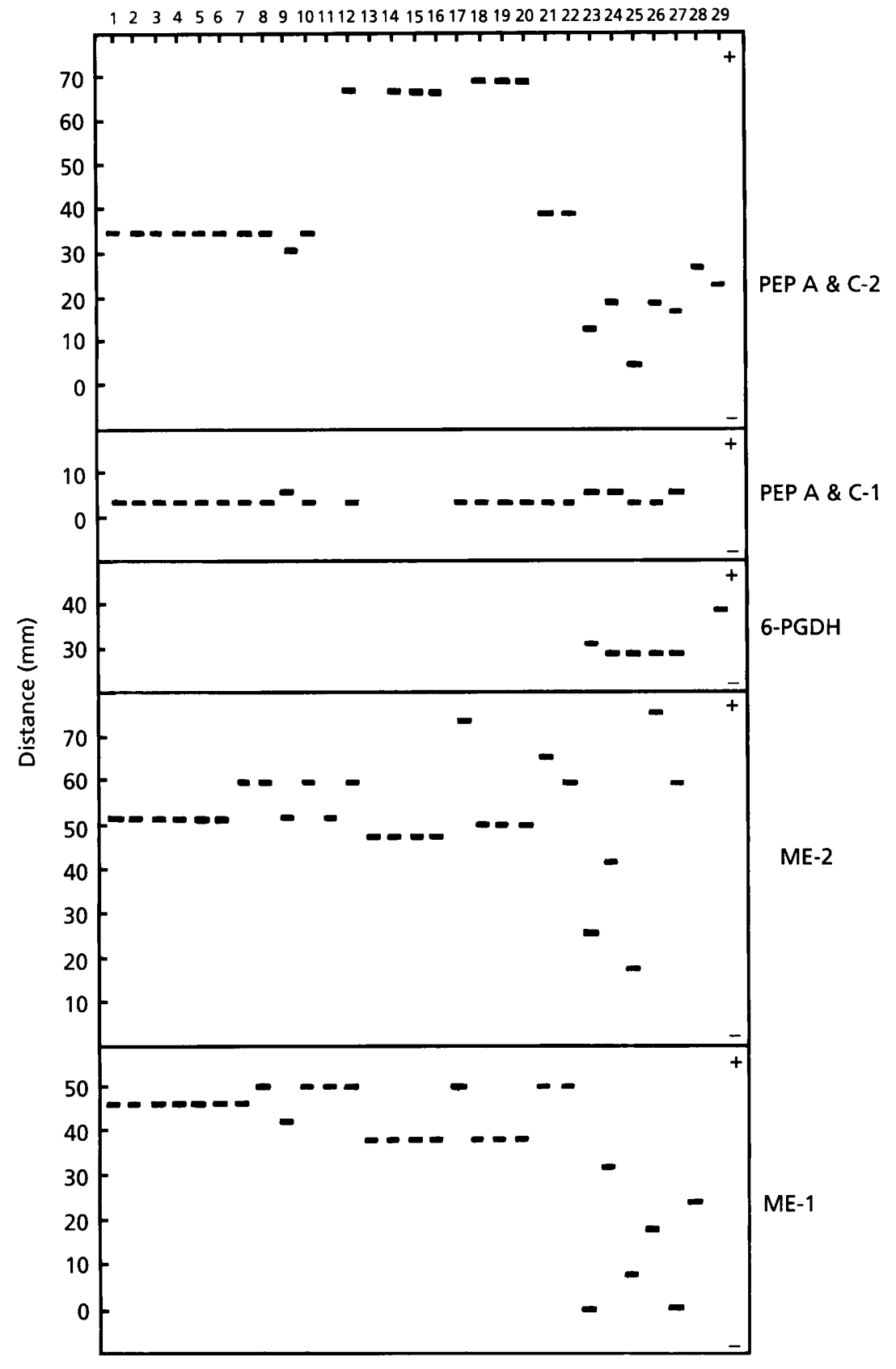

Fig. 2. Diagrammatic representation of isozyme banding patterns of PEP A \& C-1 \& $-2,6-P G D H$ and $M E-1 \&-2$. The fungi used are as listed in the legend to Fig. 1.
The phenotypes observed for all the isozyme loci are shown diagramatically in Figs 1 and 2. Representative gels of isozyme banding patterns for MDH-1 and -2 are shown photographically in Figs 3 and 4.

Nei's genetic identity or similarity $(I)$ and Nei's genetic distance $(D)$ (Table 2) were calculated to give an overall view of the relationships among the anaerobic rumen fungi and their relatedness to the aerobic chytrids. The dendrogram based on Nei's genetic identities is shown in Fig. 5. The populations were divided into two major clusters: one group containing the anaerobic rumen fungi and the other group containing the aerobic chytridiomycete fungi. Generally, the genetic identity for a particular species of rumen fungus originating from different host animals was higher than 0.80 . This indicates that the host environment did not influence the phenotype of the rumen fungus and future studies can be conducted using isolates from one host animal.

The isolate C 148, a Neocallimastix sp. originating from cattle, showed slight variation $(\bar{I}=0 \cdot 845, \bar{D}=0 \cdot 190$, Table 3) from the other $N$. variabilis isolates originating from cattle and buffalo. Although morphological characteristics of the rhizoidal systems of Neocallimastix sp. C 148 and $N$. variabillis showed some differences [rhizoids of Neocallimastix sp. C 148 were very short, compact and closely-branched below the sporangium but those of $N$. 


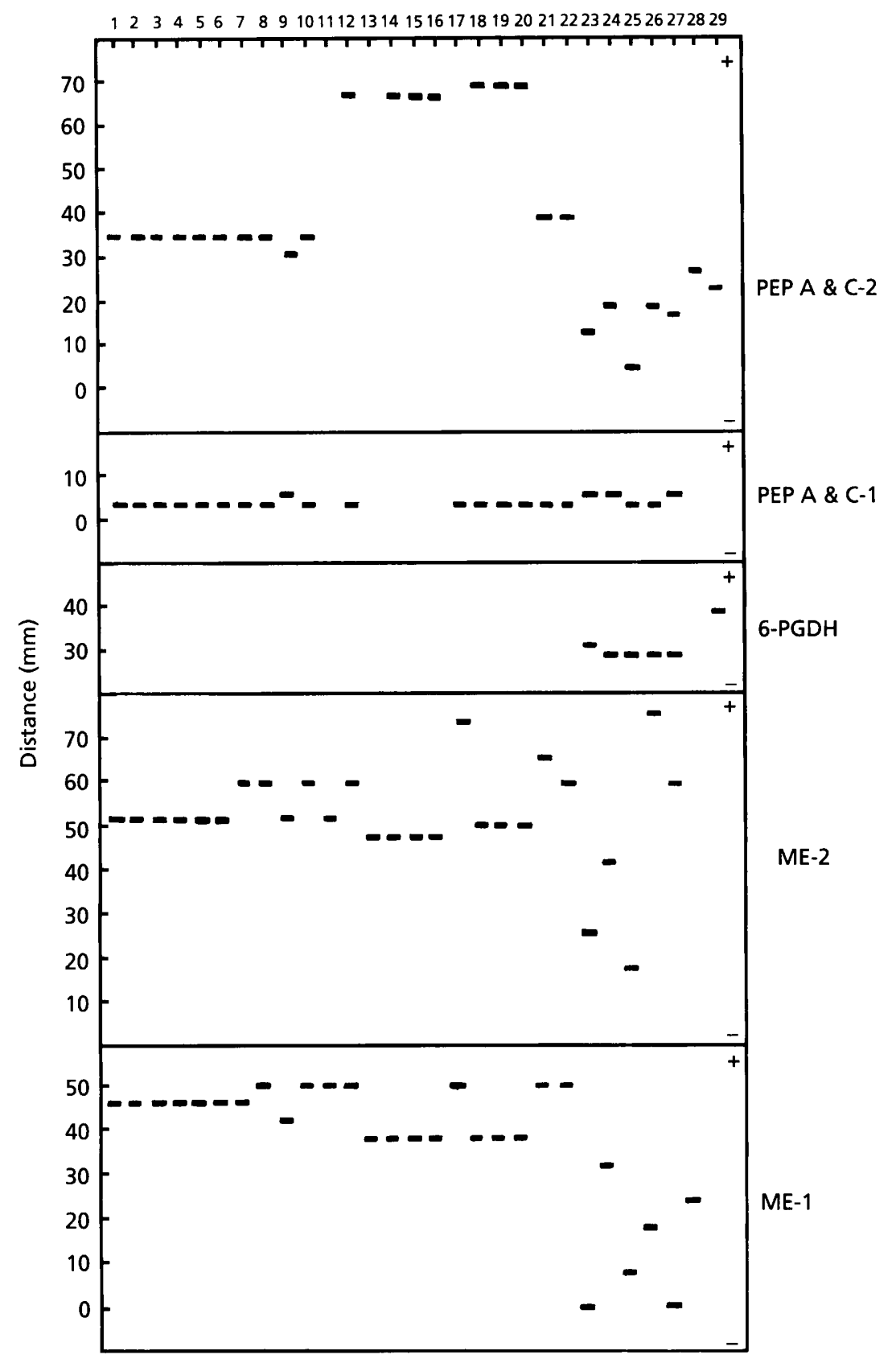

Fig. 2. Diagrammatic representation of isozyme banding patterns of PEP A \& C-1 \& $-2,6-P G D H$ and ME-1 \& -2 . The fungi used are as listed in the legend to Fig. 1.

The phenotypes observed for all the isozyme loci are shown diagramatically in Figs 1 and 2. Representative gels of isozyme banding patterns for MDH-1 and -2 are shown photographically in Figs 3 and 4.

Nei's genetic identity or similarity $(I)$ and Nei's genetic distance $(D)$ (Table 2) were calculated to give an overall view of the relationships among the anaerobic rumen fungi and their relatedness to the aerobic chytrids. The dendrogram based on Nei's genetic identities is shown in Fig. 5. The populations were divided into two major clusters: one group containing the anaerobic rumen fungi and the other group containing the aerobic chytridiomycete fungi. Generally, the genetic identity for a particular species of rumen fungus originating from different host animals was higher than $0 \cdot 80$. This indicates that the host environment did not influence the phenotype of the rumen fungus and future studies can be conducted using isolates from one host animal.

The isolate C 148, a Neocallimastix sp. originating from cattle, showed slight variation $(\bar{I}=0 \cdot 845, \bar{D}=0 \cdot 190$, Table 3) from the other $N$. variabilis isolates originating from cattle and buffalo. Although morphological characteristics of the rhizoidal systems of Neocallimastix sp. C 148 and $N$. variabillis showed some differences [rhizoids of Neocallimastix sp. C 148 were very short, compact and closely-branched below the sporangium but those of $N$. 


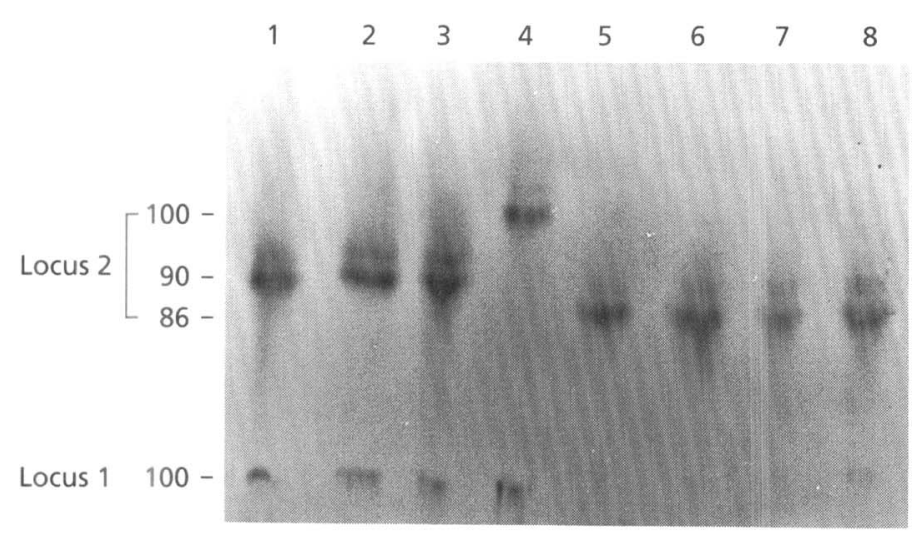

Fig. 3

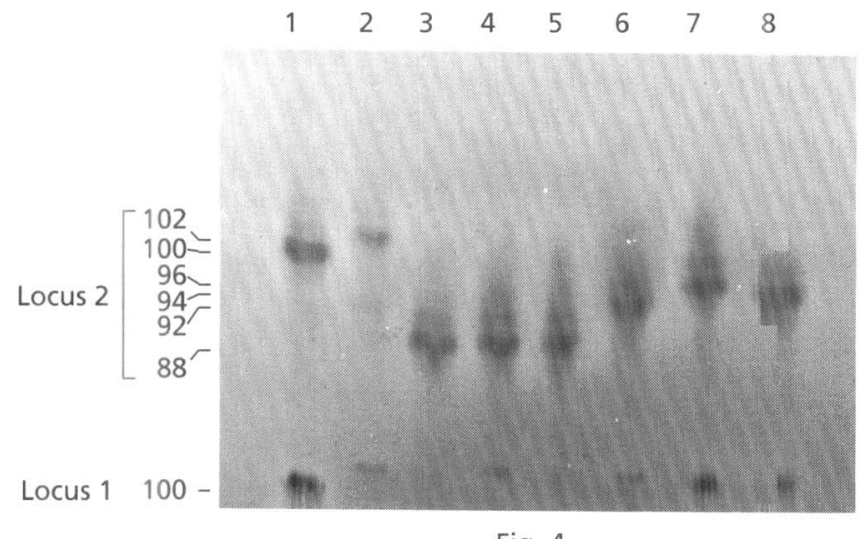

Fig. 4

Fig. 3. Typical gel with isozyme banding patterns for $\mathrm{MDH}-1$ and -2 of anaerobic rumen fungi $N$. variabilis from cattle (1 and 2), Neocalimastix sp. C148 from cattle (3), $P$. spiralis (4), $P$. minutus from deer (5), $P$. minutus from goat $(6 \& 7)$ and $P$. minutus from sheep (8). Locus 1 shows allele 100 and locus 2 shows alleles 100,90 and 86 .

Fig. 4. Typical gel with isozyme banding patterns for $\mathrm{MDH}-1$ and -2 of anaerobic rumen fungi $P$. spiralis (1), $P$. communis from goat (2 and 3), $P$. communis from sheep (4 and 5), $N$. variabilis from cattle (6), 0 . intercalaris (7) and 0 . joyonii (8). Locus 1 shows allele 100 and locus 2 shows alleles 102, 100, 96, 94,92 and 88.

variabilis were longer, less dense with a coiled main rhizoid (Ho et al., 1993a)], the genetic distance $(\bar{D}=$ $0 \cdot 190$ ) was too small to distinctly separate Neocallimastix sp. C 148 as a different species, but it may be a subspecies.

Piromyces communis and $P$. minutus fell into the same cluster (Fig. 5) indicating a close relationship $(\bar{I}=0.663, \bar{D}=$ 0.412 , Table 3). This is reflected by some of their morphological characteristics such as sporangium shape, rhizoidal system and manner of zoospore release which are quite similar. Piromyces spiralis, however, showed a more distant relationship to both $P$. communis $(\bar{I}=0.526$, $\bar{D}=0.657$, Table 3$)$ and $P$. minutus $(\bar{I}=0.416, \bar{D}=0.882$,
Table 3) (Fig. 5). This is supported by morphological studies which showed that the rhizoidal system, thallus development and sporangial wall of $P$. spiralis were quite different from those of $P$. communis and $P$. minutus (Ho $e t$ al., 1993b, c). The thallus development of $P$. spiralis is strictly endogenous, the rhizoidal system is extensive and the rhizoids are highly coiled. The neck and port of the main rhizoid of $P$. spiralis are also constricted and usually the portion below the neck is swollen and bulbous. The sporangial wall is highly evanescent and dissolution of the sporangial wall is so rapid that the zoospores are usually found in a cluster on the main rhizoid without a sporangial wall (Ho et al., 1993c).

Piromyces as a whole, showed a closer relationship to Caecomyces $(\bar{I}=0.629, \bar{D}=0.480$, Table 3$)$ than to Neocallimastix $(\bar{I}=0.361, \bar{D}=1.039$, Table 3$)$. In contrast, Dore \& Stahl (1991) using 18S rRNA sequences found Piromyces ( $P$. communis) to be more closely related to Neocallimastix (N. frontalis) than to Caecomyces (C. communis syn. Sphaeromonas communis; Gold et al., 1988). Recently, Li et al. (1993), using cladistic analysis of structural data, found that $P$. dumbonica and $C$. equi were closely related forming a cluster but $C$. communis was consistently isolated and was closer to Neocallimastix.

Orpinomyces joyonii (B 48) and O. intercalaris (C 70), although clustered together, showed a low genetic identity value $(I)$ of $0.571(D=0.560)$. This supports the contention that they are two different species based on morphological differences (Ho et al., 1994). The sporangia of $O$. intercalaris (C 70) are intercalary, produced within the hyphae in the rhizomycelium, but those of O.joyonii (B 48) are terminal, borne at the apex of sporangiophore complexes. The zoospore cyst of O. intercalaris (C 70) is also persistent and remains as a distinct structure attached to the rhizomycelium.

The dendrogram (Fig. 5) and the matrix of genetic similarity and distance coefficients (Tables 2 and 3) showed that Orpinomyces (as a whole) is more related to Piromyces $(\bar{I}=0.469, \bar{D}=0.775$, Table 3$)$ and Caecomyces $(\bar{I}=0.465, \bar{D}=0 \cdot 770$, Table 3$)$ than to Neocallimastix $(\bar{I}$ $=0 \cdot 344, \bar{D}=1 \cdot 079$, Table 3$)$. This result concurs in part with the findings of Dore \& Stahl (1991) where rRNA sequences showed Orpinomyces ( $N$. joyonii syn. O. joyonii; $\mathrm{Li}$ et al., 1991) and Piromyces (P. communis) to be closely related but they were closer to Neocallimastix ( $N$. frontalis) than to Caecomyces (C. communis syn. S. communis). However, Li \& Heath (1992), using cladistic analysis of rRNA sequences, found that the relationships between Orpinomyces, Piromyces and Neocallimastix were not clear and depended upon the algorithms used. Orpinomyces could be close to Piromyces and remote from Neocallimastix or close to Neocallimastix and remote from Piromyces. The majority of their data, however, seemed to favour, but did not strongly support, the close relationship between Orpinomyces and Piromyces. To clarify the relationships, Li et al. (1993) later used cladistic analysis of all available structural data which included morphological, ultrastructural and mitotic characteristics of the anaerobic gut fungi but again found the relationships between the three genera to be 
Table 2. Matrix of genetic similarity $(I)$ and distance $(D)$ coefficients

Numbers below diagonal: Nei (1978) unbiased genetic distance $(D)$; numbers above diagonal: Nei (1978) unbiased genetic identity or similarity $(I)$.

\begin{tabular}{|c|c|c|c|c|c|c|c|c|c|c|c|c|c|c|c|c|c|c|c|}
\hline \multirow[t]{2}{*}{ Population* } & \multicolumn{19}{|c|}{ Fungus* } \\
\hline & 1 & 2 & 3 & 4 & 5 & 6 & 7 & 8 & 9 & 10 & 11 & 12 & 13 & 14 & 15 & 16 & 17 & 18 & 19 \\
\hline 1 & & $0-936$ & 0.754 & 0.439 & 0.367 & 0.295 & 0.283 & $0 \cdot 283$ & 0.264 & 0.358 & 0.264 & 0.283 & $0 \cdot 264$ & 0.151 & $0 \cdot 170$ & 0.283 & $0 \cdot 170$ & 0.151 & 0.132 \\
\hline 2 & 0.067 & & 0.908 & 0.525 & 0.448 & 0.379 & 0.377 & $0 \cdot 360$ & 0.360 & 0.428 & 0.394 & 0.411 & 0.326 & 0.154 & $0 \cdot 188$ & 0.291 & $0 \cdot 188$ & $0 \cdot 206$ & 0.171 \\
\hline 3 & 0.283 & 0.096 & & 0.437 & 0.366 & $0 \cdot 309$ & 0.286 & $0 \cdot 286$ & 0.429 & 0.357 & 0.357 & 0.357 & 0.286 & 0.143 & 0.143 & 0.286 & 0.143 & $0 \cdot 214$ & 0.143 \\
\hline 4 & 0.824 & 0.645 & 0.827 & & 0.874 & 0.638 & 0.656 & 0.656 & 0.612 & 0.744 & 0.525 & 0.612 & 0.525 & 0.437 & 0.481 & 0.437 & 0.481 & 0.437 & 0.350 \\
\hline 5 & 1.003 & 0.804 & 1.005 & 0.135 & & 0.672 & 0.695 & 0.659 & 0.439 & 0.586 & 0.476 & 0.586 & 0.439 & 0.293 & $0 \cdot 366$ & $0 \cdot 366$ & $0-366$ & 0.293 & 0.220 \\
\hline 6 & $1 \cdot 220$ & 0.969 & $1 \cdot 176$ & 0.450 & 0.398 & & 0.926 & 0.887 & 0.463 & 0.656 & 0.386 & 0.540 & 0.386 & 0.386 & 0.386 & $0 \cdot 347$ & $0 \cdot 386$ & $0-309$ & 0.347 \\
\hline 7 & 1.263 & 0.976 & $1 \cdot 253$ & 0.421 & 0.363 & 0.077 & & 0.786 & 0.429 & 0.643 & 0.429 & 0.500 & 0.357 & 0.357 & 0.357 & 0.286 & 0.357 & 0.286 & 0.286 \\
\hline 8 & 1.263 & 1.022 & $1 \cdot 253$ & 0.421 & 0.417 & $0 \cdot 120$ & $0 \cdot 241$ & & 0.357 & 0.714 & 0.357 & 0.500 & 0.357 & 0.357 & 0.357 & 0.286 & 0.357 & 0.286 & $0 \cdot 286$ \\
\hline 9 & 1.332 & 1.022 & 0.847 & 0.490 & 0.823 & $0 \cdot 770$ & 0.847 & 1.030 & & 0.429 & 0.357 & 0.357 & $0 \cdot 286$ & 0.286 & $0 \cdot 214$ & 0.286 & 0.214 & 0.357 & 0.286 \\
\hline 10 & 1.027 & 0.848 & 1.030 & 0.296 & 0.535 & 0.422 & 0.442 & 0.336 & 0.847 & & 0.429 & 0.500 & 0.357 & 0.357 & 0.357 & 0.286 & 0.357 & 0.286 & $0 \cdot 286$ \\
\hline 11 & $1 \cdot 332$ & 0.931 & 1.030 & 0.645 & 0.743 & 0.953 & 0.847 & $1 \cdot 030$ & 1.030 & 0.847 & & 0.571 & 0.286 & 0.071 & 0.143 & 0.286 & 0.143 & 0.071 & 0.241 \\
\hline 12 & 1.263 & 0.889 & 1.030 & 0.490 & 0.535 & $0-616$ & 0.693 & 0.693 & 1.030 & 0.693 & 0.560 & & $0-429$ & 0.214 & 0.286 & 0.286 & 0.286 & 0.286 & 0.286 \\
\hline 13 & 1.332 & $1 \cdot 122$ & 1.253 & 0.645 & 0.823 & 0.953 & $1 \cdot 030$ & 1.030 & 1.253 & 1.030 & 1.253 & 0.847 & & 0.357 & 0.357 & 0.500 & 0.429 & 0.429 & $0 \cdot 286$ \\
\hline 14 & 1.892 & 1.870 & 1.946 & 0.827 & 1.228 & 0.953 & 1.030 & 1.030 & 1.253 & 1.030 & 2.639 & 1.540 & 1.030 & & 0.429 & 0.429 & 0.429 & 0.571 & 0.643 \\
\hline 15 & 1.774 & 1.669 & 1.946 & 0.732 & 1.005 & 0.953 & $1 \cdot 030$ & $1 \cdot 030$ & 1.540 & 1.030 & 1.946 & 1.253 & 1.030 & 0.847 & & 0.357 & 0.571 & 0.571 & 0.286 \\
\hline 16 & 1.263 & $1 \cdot 234$ & $1 \cdot 253$ & 0.827 & $1 \cdot 005$ & 1.058 & $1 \cdot 253$ & $1 \cdot 253$ & $1 \cdot 253$ & 1.253 & 1.253 & $1 \cdot 253$ & 0.693 & 0.847 & $1 \cdot 030$ & & 0.571 & $0-429$ & 0.357 \\
\hline 17 & 1.774 & 1.669 & 1.946 & 0.732 & 1.005 & 0.953 & 1.030 & 1.030 & 1.540 & $1 \cdot 030$ & 1.946 & $1 \cdot 253$ & 0.847 & 0.847 & 0.560 & 0.560 & & 0.500 & 0.286 \\
\hline 18 & 1.892 & 1.582 & 1.540 & 0.827 & 1.228 & 1.176 & 1.253 & $1 \cdot 253$ & 1.030 & $1 \cdot 253$ & 2.639 & $1 \cdot 253$ & 0.847 & 0.560 & 0.560 & 0.847 & 0.693 & & 0.429 \\
\hline 19 & 2.026 & 1.764 & 1.946 & 1.050 & 1.516 & $1 \cdot 058$ & $1 \cdot 253$ & 1.253 & 1.253 & 1.253 & 1.540 & $1 \cdot 253$ & 1.253 & $0 \cdot 442$ & 1.253 & 1.030 & 1.253 & 0.847 & \\
\hline
\end{tabular}

* 1, N. variabilis (buffalo); 2, N. variabilis (cattle); 3 , Neocallimastix sp. C148; 4, P. communis (goat); 5 , P. communis (sheep); 6, P. minutus (goat); 7, P. minutus (sheep); 8, P. minutus (deer); 9, P. spiralis (goat); 10, Caecomyces sp. (buffalo); 11, O. joyonii (buffalo); 12, O. intercalaris C70 (cattle); 13, S. plurigibbosus BR 33; 14, Chytridium confervae BR 97; 15, S. punctatus BR 117;16, R. rosea BR 60; 17, S. acuminatus BR 62; 18, R. rosea BR 186; 19, Catenaria anguillulae BR 105.

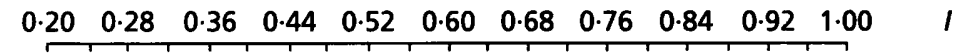

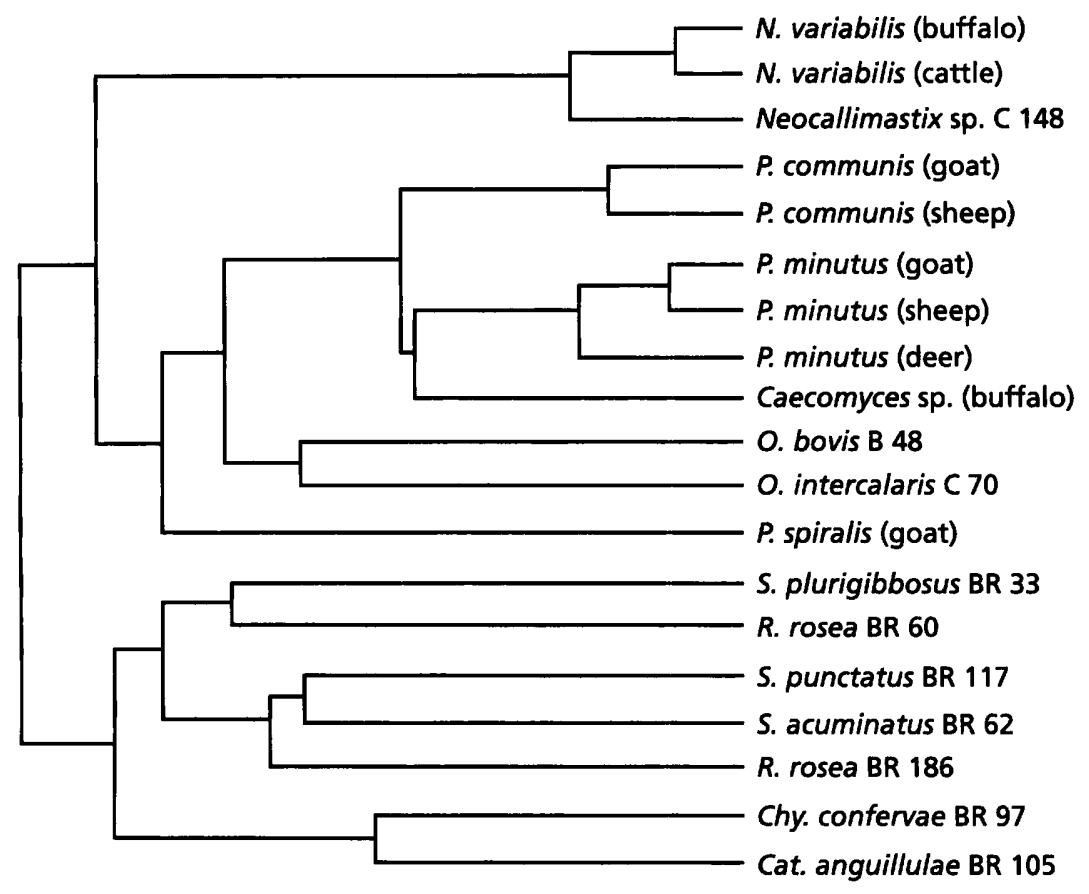

$\begin{array}{llllllllllll}0.20 & 0.28 & 0.36 & 0.44 & 0.52 & 0.60 & 0.68 & 0.76 & 0.84 & 0.92 & 1.00\end{array}$
Fig. 5. Dendrogram showing the genetic relationships among the anaerobic rumen fungi and the aerobic chytridiomycete fungi. inconsistent depending on the set of characteristics used or the outgroup chosen. Orpinomyces could be distant from Piromyces and close to or distant from Neocallimastix.
Based on the genetic identity data of all the anaerobic rumen fungi studied, $P$. communis seems to be most related to the aerobic chytrids, followed by $P$. minutus, Caecomyces 
Table 3. Relationships among the anaerobic rumen fungi based on the mean cumulative similarity $(\bar{l})$ or distance $(\bar{D})$ coefficients

\begin{tabular}{|lcc|}
\hline Relationship between fungi & $\overline{\mathbf{I}}$ & $\overline{\mathbf{D}} \boldsymbol{}$ \\
\hline N. rariabilis and Neocallimastix & $0 \cdot 845$ & $0 \cdot 190$ \\
sp. C148 & & \\
P. minutus and P. communis & 0.663 & 0.412 \\
P. spiralis and P. communis & 0.526 & 0.657 \\
P. spiralis and P. minutus & $0 \cdot 416$ & 0.882 \\
Piromyces and Caecomyces & $0 \cdot 629$ & 0.480 \\
Piromyces and Neocallimastix & 0.361 & 1.039 \\
Caecomyces and Neocallimastix & 0.381 & 0.968 \\
O. joyonii and & 0.571 & 0.560 \\
O. intercalaris C70 & & \\
Orpinomyces and Piromyces & 0.469 & 0.775 \\
Orpinomyces and Caecomyces & 0.465 & 0.770 \\
Orpinomyces and Neocallimastix & 0.344 & 1.0792 \\
\hline
\end{tabular}

* Higher value indicates closer relationship.

† Higher value indicates greater genetic distance and more remote relationship.

Table 4. The relatedness of different species of anaerobic rumen fungi to the aerobic chytridiomycete fungi based on the mean cumulative similarity coefficients $(\bar{l})$

\begin{tabular}{|lc|}
\hline Species & $\overline{\boldsymbol{I}}^{*}$ \\
\hline Piromyces communis & $0 \cdot 392$ \\
Piromyces minutus & $0 \cdot 339$ \\
Caecomyces sp. & $0 \cdot 327$ \\
Orpinomyces intercalaris C70 & $0 \cdot 296$ \\
Piromyces spiralis & $0 \cdot 276$ \\
Neocallimastix variabilis & $0 \cdot 203$ \\
Neocallimastix sp. C148 & $0 \cdot 194$ \\
Orpinomyces joyonii & $0 \cdot 177$ \\
\hline
\end{tabular}

* Higher value indicates closer relationship.

sp., O. intercalaris C 70, P. spiralis, N. variabilis, Neocallimastix sp. C 148 and finally O. joyonii C 48 (Table 4).

The dendrogram based on Nei's genetic identity (Fig. 5) shows that the anaerobic rumen fungi form a distinct cluster indicating a monophyletic group. They also form a separate cluster from the Spizellomycetales, indicating that as a group they are distinctive from the Spizellomycetales. This supports the establishment of a new order, the Neocallimastigales, by $\mathrm{Li}$ et al. (1993) to accommodate the anaerobic gut fungi. Other workers (Heath \& Bauchop, 1985; Munn et al., 1987) have also observed distinct differences in some of the mitotic and ultrastructural characteristics of the anaerobic gut fungi and have suggested the establishment of a new order for the anaerobic gut fungi.

In this study, isozymes were used to determine the genetic relationships among existing species of anaerobic rumen fungi and aerobic chytrids. For this purpose, Nei's genetic distances (Nei, 1978) based on gene frequencies and the UPGMA dendrogram (Sneath \& Sokal, 1973) are suitable. Cladistic analysis has been used and recommended for the study of phylogenetic relationships among species of fungi but this approach is not used here as we are not proposing a phylogenetic tree, nor are we determining the evolutionary relationships between species. Phylogenetic systematics is a taxonomic system based on the theory of descent. When phylogenetic relationships are developed using the principle of parsimony (minimizing numbers of assumptions), a pattern of characters may be explained by a number of conflicting relationships or trees. In these cases, a further analytical tool is needed for rejecting some of them. It is in these cases that the cladistic analysis is frequently used. When the principle behind cladistic analysis is scrutinized, it has been noted that if the cladistic type of outgroup analysis can be applied unambiguously, it coincides with the encaptic pattern achieved by the parsimony analysis and is not required (Lorenzen, 1993). When there is a need for further analysis to accept the correct hypothesis(es) from varying results from parsimony analysis, cladistic analysis is useless or is misleading. Depending on the outgroup, different hypotheses are suggested (Lorenzen, 1993).

Nei's genetic distance gives the measure of the amount of genetic divergence between populations (species) or subpopulations (subspecies). It accomplishes a hierarchical grouping of populations based on normalized identity. The identity here refers to how 'indistinguishable' the populations are and not to 'identify by descent'.

In conclusion, this work demonstrates the usefulness of isozyme characteristics for studying the genetic relationships among the anaerobic rumen fungi and their relationships with other aerobic chytridiomycete fungi. Isozyme characteristics can be used as additional taxonomic criteria in the classification of the anaerobic rumen fungi. The genetic data from this study show that: (i) the anaerobic rumen fungi form a cluster, indicating a monophyletic group distinctly separated from the aerobic chytridiomycete fungi; (ii) they are separate from the Spizellomycetales; (iii) $P$. communis and $P$. minutus are more related to each other than to P. spiralis; (iv) Piromyces and Caecomyces are closer to each other than either is to Neocallimastix; (v) Orpinomyces is more related to Piromyces than to Neocallimastix; and (vi) O. intercalaris C 70 is distinctly different from $O$. joyonii, indicating that it is a new species.

\section{REFERENCES}

Barr, D. J. S. (1980). An outline for the classification of the Chytridiales, and for a new order, the Spizellomycetales. Can J Bot 58, 2380-2394.

Barr, D. J. S. (1984). The classification of Spizellomyces, Gaetneriomyces, Triparticalar and Kochiomyces (Spizellomycetales, Chytridiomycetes). Can J Bot 62, 1171-1201.

Barr, D. J. S., Kudo, H., Jakober, K. D. \& Cheng, K.-J. (1989). Morphology and development of rumen fungi Neocallimastix sp., 
Piromyces communis and Orpinomyces bovis gen. nov., sp. nov. Can J Bot 67, 2815-2824.

Bowman, B., Taylor, J. W., Brownlee, A. G., Lee, J., Lu, S. D. \& White, T. J. (1992). Molecular evolution of the fungi: relationship of the Basidiomycetes, Ascomycetes and Chytridiomycetes. Mol Biol Evol 9, 285-296.

Breton, A., Bernalier, A., Dusser, M., Fonty, G., Gaillard-Martinie, B. \& Guillot, J. (1990). Anaeromyces mucronatus gen. nov., sp. nov. A. new strictly anaerobic rumen fungus with polycentric thallus. FEMS Microbiol Lett 70, 177-182.

Breton, A., Dusser, M., Gaillard-Martinie, B., Guillot, J., Millet, L. \& Prensier, G. (1991). Piromyces rbizinflata sp. nov., a strictly anaerobic fungus from faeces of the Saharian ass : a morphological, metabolic and ultrastructural study. FEMS Microbiol Lett 82, 1-8.

Dore, J. \& Stahl, D. A. (1991). Phylogeny of anaerobic rumen Chytridiomycetes inferred from small subunit ribosomal RNA sequence comparisons. Can J Bot 69, 1964-1971.

Gleason, F. H. \& Gordon, G. L. R. (1990). Lactate dehydrogenase in obligately anaerobic Chytridiomyces from the rumen. Mycologia $\mathbf{8 2}$, 261-263.

Gold, J. J., Heath, I. B. \& Bauchop, T. (1988). Ultrastructural description of a new chytrid genus of caecum anaerobe, Caecomyces equi gen. nov., sp. nov., assigned to the Neocallimasticaceae. BioSystems 21, 403-415.

Harris, H. \& Hopkinson, D. A. (1976). Handbook of Enzyme Electrophoresis in Human Genetics. Amsterdam: North Holland Publishing Company.

Heath, I. B. \& Bauchop, T. (1985). Mitosis and the phylogeny of the genus Neocallimastix. Can J Bot 63, 1595-1604.

Heath, I. B., Bauchop, T. \& Skipp, R. A. (1983). Assignment of the rumen anaerobe Neocallimastix frontalis to the Spizellomycetales (Chytridiomycetes) on the basis of its polyflagellate zoospore ultrastructure. Can J Bot 61, 295-307.

Ho, Y. W. \& Bauchop, T. (1991). Morphology of three polycentric rumen fungi and description of a procedure for the induction of zoosporogenesis and release of zoospores in cultures. J Gen Microbiol 137, $213-217$.

Ho, Y. W., Abdullah, N. \& Jalaludin, S. (1994). Orpinomyces intercalaris, a new species of polycentric anaerobic rumen fungus from catle. Mycotaxon 50, 139-150.

Ho, Y. W., Bauchop, T., Abdullah, N. \& Jalaludin, S. (1990). Ruminomyces elegans gen. et sp. nov., a polycentric anaerobic rumen fungus from cattle. Mycotaxon 38, 397-405.

Ho, Y. W., Barr, D. J. S., Abdullah, N., Jalaludin, S. \& Kudo, H. (1993a). Neocallimastix variabilis, a new species of anaerobic fungus from the rumen of cattle. Mycotaxon 46, 241-258.

Ho, Y. W., Barr, D. J. S., Abdullah, N., Jalaludin, S. \& Kudo, H. (1993b). A new species of Piromyces from the rumen of deer in Malaysia. Mycotaxon 47, 285-293.

Ho, Y. W., Barr, D. J. S., Abdullah, N., Jalaludin, S. \& Kudo, H. (1993c). Piromyces spiralis, a new species of anaerobic fungus from the rumen of goat. Mycotaxon 48, 59-68.

Hungate, R. F. (1969). A roll tube method for cultivation of strict anaerobes. Methods Microbiol 3B, 117-132.

Index of Fungi Supplement (Family Names) (1989). Compiled by D. L. Hawksworth and J. C. David. Wallingford: CAB International.

Li, J. \& Heath, I. B. (1992). The phylogenetic relationships of the anaerobic chytridiomycetous gut fungi (Neocallimasticaceae) and the Chytridiomycota. I. Cladistic analysis of rRNA sequences. Can $J$ Bot 70, 1738-1746.

Li, J., Heath, I. B. \& Bauchop, T. (1990). Piromyces mae and Piromyces dumbonica, two new species of uniflagellate anaerobic chytridiomycete fungi from the hindgut of the horse and elephant. Can J Bot 68, 1021-1033.

Li, J., Heath, I. B. \& Cheng, K.-J. (1991). The development and zoospore ultrastructure of a polycentric chytridiomycete gut fungus, Orpinomyces joyonii comb. nov. Can J Bot 69, 580-589.

Li, J., Heath, I. B. \& Packer, L. (1993). The phylogenetic relationships of the anaerobic chytridiomycetous gut fungi (Neocallimasticaceae) and the Chytridiomycota. II. Cladistic analysis of structural data and description of Neocallimasticales ord. nov. Can J Bot 71, 393-407.

Lorenzen, S. (1993). The role of parsimony, outgroup analysis and theory of evolution in phylogenetic systematics. Z Zool Syst Evolutionsforsch 31, 1-20.

Munn, E. A., Greenwood, C. A. \& Orpin, C. G. (1987). Organization of the kinetosomes and associated structures of zoospores of the rumen chytridiomycete Neocallimastix patriciarum. Can J Bot 65, 456-465.

Nei, M. (1978). Estimation of average heterozygosity and genetic distance from a small number of individuals. Genetics 89, 583-590.

Orpin, C. G. (1975). Studies on the rumen flagellate Neocallimastix frontalis. J Gen Microbiol 91, 249-262.

Sneath, P. H. A. \& Sokal, R. R. (1973). Numerical Taxonomy. San Francisco: W. H. Freeman.

Steiner, W. W. M. \& Joslyn, D. J. (1979). Electrophoretic techniques for the genetic study of mosquitoes. Mosq News 39, 35-54.

Swofford, D. L. \& Selander, R. B. (1989). вiosYs-1: a computer program for analysis of allele variation in population genetics and biochemical systematics (Release 1.7). Champaign, IL: Illinois Natural History Survey.

Tan, S. G. \& Teng, Y. S. (1979). Human saliva as a source of biochemical genetic markers I. Techniques. Hum Hered 29, 69-76.

Vallejos, C. E. (1983). Enzyme activity staining. In Isoenzymes in Plant Genetics and Breeding. Edited by S. D. Tanskley \& T. J. Orton. Amsterdam: Elsevier Science Publishers.

Received 4 October 1993; revised 13 December 1993; accepted 17 January 1994. 